\title{
The inflammatory milieu within the pancreatic cancer microenvironment correlates with clinicopathologic parameters, chemoresistance and survival
}

\author{
Daniel Delitto ${ }^{1}$, Brian S. Black', Heather L. Sorenson², Andrea E. Knowlton², Ryan M. Thomas ${ }^{1,3}$, George A. Sarosi ${ }^{1,3}$, \\ Lyle L. Moldawer', Kevin E. Behrns ${ }^{1}$, Chen Liư ${ }^{4}$, Thomas J. George ${ }^{5}$, Jose G. Trevino ${ }^{1}$, Shannon M. Wallet ${ }^{2}$ \\ and Steven J. Hughes ${ }^{1 *}$
}

\begin{abstract}
Background: The tumor microenvironment impacts pancreatic cancer (PC) development, progression and metastasis. How intratumoral inflammatory mediators modulate this biology remains poorly understood. We hypothesized that the inflammatory milieu within the PC microenvironment would correlate with clinicopathologic findings and survival.

Methods: Pancreatic specimens from normal pancreas $(n=6)$, chronic pancreatitis $(n=9)$ and pancreatic adenocarcinoma $(n=36)$ were homogenized immediately upon resection. Homogenates were subjected to multiplex analysis of 41 inflammatory mediators.

Results: Twenty-three mediators were significantly elevated in adenocarcinoma specimens compared to nonmalignant controls. Increased intratumoral IL-8 concentrations associated with larger tumors $(P=.045)$ and poor differentiation $(P=.038)$; the administration of neoadjuvant chemotherapy associated with reduced IL-8 concentrations $(P=.003)$. Neoadjuvant therapy was also associated with elevated concentrations of Flt-3 $L(P=.005)$. Elevated levels of pro-inflammatory cytokines IL-1 $\beta(P=.017)$ and TNFa $(P=.033)$ were associated with a poor histopathologic response to neoadjuvant therapy. Elevated concentrations of G-CSF $(P=.016)$ and PDGF-AA $(P=.012)$ correlated with reduced overall survival. Conversely, elevated concentrations of FGF-2 $(P=.038)$, TNFa $(P=.031)$ and MIP-1a $(P=.036)$ were associated with prolonged survival.
\end{abstract}

Conclusion: The pancreatic cancer microenvironment harbors a unique inflammatory milieu with potential diagnostic and prognostic value.

Keywords: Inflammation, Cytokines, Chemokines, Growth factors, Pancreatic cancer, Tumor microenvironment

\section{Background}

Pancreatic adenocarcinoma (PC) is the fourth leading cause of cancer deaths in the United States, due in part to nearly universal resistance to cytotoxic chemotherapy. Gemcitabine-based therapies achieve clinical benefit in approximately $24 \%$ of patients with PC [1], but the

\footnotetext{
* Correspondence: Steven.Hughes@surgery.ufl.edu

'Department of Surgery, College of Medicine, University of Florida Health Science Center, Room 6116, Shands Hospital, 1600 SW Archer Rd, Gainesville, FL 32610, USA

Full list of author information is available at the end of the article
}

overall survival advantages are sobering, ranging from a few weeks to months $[1-3]$. Complete surgical resection offers patients with $\mathrm{PC}$ the greatest survival benefit. However, this is achievable in fewer than $20 \%$ of patients presenting with $\mathrm{PC}[4]$. As a result, PC is projected to be the second leading cause of cancer deaths by 2030 [5]. There is a tremendous need to discover novel biomarker (s) or panels of biomarkers that can aid in detecting PC earlier, improving prognostic evaluation and predicting response to chemotherapy.

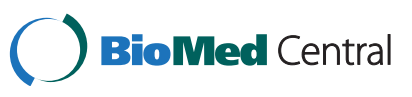

(c) 2015 Delitto et al. Open Access This article is distributed under the terms of the Creative Commons Attribution 4.0 International License (http://creativecommons.org/licenses/by/4.0/), which permits unrestricted use, distribution, and reproduction in any medium, provided you give appropriate credit to the original author(s) and the source, provide a link to the Creative Commons license, and indicate if changes were made. The Creative Commons Public Domain Dedication waiver (http://creativecommons.org/publicdomain/zero/1.0/) applies to the data made available in this article, unless otherwise stated. 
Inflammation within the PC microenvironment has been mechanistically linked to tumor progression and chemoresistance through NF-kB, IL-6, toll-like receptor and TGF- $\beta$ signaling pathways $[6-10]$. However, the diagnostic and prognostic value of the inflammatory milieu within the PC microenvironment remains essentially undefined. While survival gains from immune cell infiltration into the tumor microenvironment have been conclusively demonstrated in colorectal and ovarian cancer [11-13], similar investigations have not yielded consistent results in PC $[14,15]$. Patients with chronic pancreatitis are 5-15 times more likely to develop PC [16] and insights into the association between inflammation and PC stems from investigations of chronic pancreatitis. Potential environmental sequelae of pancreatitis such as hypoxia, the presence of reactive oxygen species, and acidosis may influence the development of PC [17]. Additionally, numerous soluble mediators, including TNF- $\alpha$ [18], TGF- $\alpha$ [19], TGF- $\beta$ [20], IL-1 $\beta$ [21], IL-1 $\alpha$ [22], IL-6 [23, 24], IL-8 [25], VEGF [26], and others have been implicated in PC carcinogenesis, tumor progression, and treatment resistance. However, the relationship between the inflammatory milieu and the spectrum of disease from normal pancreas to pancreatitis to pancreatic cancer has not yet been characterized. Therefore, the translational relevance of the microenvironmental inflammatory milieu to PC development and progression remains speculative.

We examined the inflammatory milieu present in the PC microenvironment from 36 freshly resected tumor specimens using a forty-one-item panel of cytokines, chemokines and growth factors to test the hypothesis that expression levels of these mediators harbor diagnostic and prognostic value. We first compared the inflammatory milieu of PC to that of pancreatitis $(n=9)$ and normal pancreas $(n=6)$. Inflammatory mediators were further evaluated in relation to prognostic clinicopathologic parameters, administration of neoadjuvant therapy, treatment resistance and patient survival. These data bring the field one step closer to the identification of biomarker panels that can aid in detecting disease earlier and classifying patients with respect to response to chemotherapy and most importantly, prognosis.

\section{Materials and methods Patient cohorts}

A prospectively maintained database approved by the Institutional Review Board at the University of Florida (353-2007) was utilized for sample selection. Written informed consent was obtained from all participants. In total, 51 samples were included in this study. Using pathologically verified diagnoses, samples were placed into one of three experimental groups: normal pancreas $(n=6)$, chronic pancreatitis $(n=9)$ and pancreatic carcinoma $(n=36)$. Indications for resection of 'normal' pancreata included duodenal adenomas $(\mathrm{n}=3)$, remotely located neuroendocrine tumors $(n=2)$ and a ductal squamoid cyst $(n=1)$. Of the 36 patients with pathologically confirmed pancreatic adenocarcinoma, all underwent resection with curative intent, 10 whom completed gemcitabine/abraxane-based neoadjuvant chemotherapy. Pathologic response to neoadjuvant chemotherapy was graded by clinical pathologists upon resection using a validated scale [27]. Briefly, histopathologic response to neoadjuvant therapy was broadly grouped into complete ( $>90 \%$ of tumor cells destroyed), moderate (10-90\% of tumor cells destroyed) and poor $(<10 \%$ of tumor cells destroyed). All 36 patients had at least 6 months of clinical follow-up for survival analysis.

\section{Pancreatic tissue harvest}

Resected pancreatic tissue was immediately weighed and placed in cell lysis buffer (Cell Signaling Technologies, Danvers, MA) with a protease inhibitor cocktail (SigmaAldrich, St. Louis, MO). Immediately adjacent tissues were preserved in formalin for histologic verification of pathology. Tissues were dissociated mechanically and further homogenized using the FastPrep-24 system according to the manufacturer's protocol (MP Biomedicals, Santa Ana, CA). Homogenates were stored at $-80{ }^{\circ} \mathrm{C}$ until soluble mediator analysis could be performed.

\section{Soluble mediator analysis}

Homogenates were then probed for soluble mediators using the Milliplex ${ }^{\circ}$ Premixed 41-Plex Immunology Multiplex Assay (Merck Millipore, Darmstadt, Germany) according to the manufacturer's protocol. Specifically, supernatants from tissue homogenates were incubated in filter bottom microtiter plates (EMD Millipore, San Jose, CA) with beads coated with primary antibodies overnight at 4C. After washing, PE- conjugated anti-cytokine antibodies were added and incubated for additional $2 \mathrm{~h}$ at room temperature. Following washing, data was acquired on a Luminex 200 (EMD Millipore, San Jose, CA) and analyzed with Milliplex Software (EMD Millipore, San Jose, CA). Concentrations were quantified using a standard curve and 5 parameter logistics to determine $\mathrm{pg} / \mathrm{mL}$ concentrations.

All cytokine concentrations were normalized to total protein concentrations using detergent compatible protein quantification (Bio-Rad, Hercules, CA). Soluble mediator concentrations were then converted to $\mathrm{pg} / \mathrm{mg}$ of tissue as follows: $\mathrm{pg} / \mathrm{ml}$ divided by $\mathrm{mg} / \mathrm{ml}$ of total protein.

\section{Statistical analysis}

All statistical analysis was performed using SPSS version 22.0 (IBM SPSS Statistics for Windows; IBM Corp). For each normalized tissue cytokine concentration, represented in picograms per milligram of total protein ( $\mathrm{pg} / \mathrm{mg}$ protein), 
normality was assessed using the Shapiro-Wilk test. Since all normalized cytokine concentrations did not display normal distributions $(P<0.05)$, non-parametric testing was employed to evaluate differences. In this manner, the Mann Whitney $U$ test was incorporated for binomial categorical variables, and $P<0.05$ was considered statistically significant. Additionally, Spearman's rank correlation coefficients were employed to determine significant associations between continuous variables. Overall survival was calculated using the following formula: Number of days from date of surgery to death or the date of last followup, whichever came first, divided by 365.25 (accounting for leap years), multiplied by 12 to obtain the time in months. Kaplan-Meier survival curves were generated using median intratumoral concentration to dichotomize PC specimens into cytokine high $_{\text {and cyto- }}$ kine $_{\text {low }}$ groups. The log-rank (Mantel-Cox) test was used to evaluate statistical significance. Additionally, a univariate Cox proportional hazards model was used to generate hazard ratios. Each soluble mediator was then incorporated into a multivariate proportional hazards model with the degree of lymphatic metastasis, as this was the only clinicopathologic parameter demonstrating a significant correlation with survival $(P<.05)$ on univariate analysis.

\section{Results}

\section{Pancreatic adenocarcinoma has a distinct intratumoral inflammatory milieu}

Establishing the diagnosis of PC remains a significant clinical problem that delays initiation of therapy, impacts enrollment in clinical trials, and mandates that patients undergo major surgical procedures in the absence of definitive findings. In order to determine whether the intratumoral inflammatory milieu may have diagnostic value, we measured the concentrations of 41 cytokines, chemokines and growth factors in 51 freshly homogenized pancreatic surgical samples. We found no significant differences in any of the normalized cytokine concentrations when comparing normal pancreatic tissue $(n=6)$ to that of chronic pancreatitis $(n=9)$. Thus, pairwise comparisons between nonmalignant tissue $(n=15)$ and adenocarcinoma $(n=36)$ as well as between pancreatitis alone $(n=9)$ and adenocarcinoma $(n=36)$ were performed (Table 1$)$. Of the 41-protein-panel of cytokines, chemokines and growth factors evaluated, the concentrations of 23 emerged as significantly higher in pancreatic cancer compared to nonmalignant tissue. The most significant differences $(P<.001)$ were observed for Eotaxin, IP-10, MCP1, MCP3, MDC, IL$1 \alpha$, IL-1RA, IL-7, and IL-8. Interestingly, all but 3 (FGF-2, RANTES and IL1 $\beta$ ) of the 23 mediators that emerged as significant when comparing pancreatic cancer to nonmalignant tissue also emerged as significant when comparing pancreatic cancer to pancreatitis. Together these data suggest that pancreatic adenocarcinoma has a distinct inflammatory milieu when compared to that of nonmalignant pancreatic tissues, including that of chronic pancreatitis.

\section{Elements of the intratumoral inflammatory milieu strongly associate with the administration of neoadjuvant cytotoxic chemotherapy}

Dynamic changes accompanying the administration of cytotoxic chemotherapy within the PC microenvironment remain poorly described. In order to determine whether differences within intratumoral inflammatory milieu associate with the administration of cytotoxic chemotherapy, patients were dichotomized into groups based on the administration of neoadjuvant therapy. Indeed, significantly lower levels of intratumoral IL-8 were observed in PC specimens from patients treated with neoadjuvant gemcitabinebased regimens compared to those from treatment naïve patients (median concentration $1129 \mathrm{pg} / \mathrm{mg}$ protein vs. $114 \mathrm{pg} / \mathrm{mg}$ protein; $P=.003$ ) (Table 2 ). Conversely, high intratumoral concentrations of Flt-3 L and IL-2 correlated with the administration of neoadjuvant chemotherapy. Together these data suggest that the administration of cytotoxic chemotherapy alters the inflammatory microenvironment in PC.

Poor histopathologic response to neoadjuvant chemotherapy appears to associate with poor clinical outcomes, although this phenomenon continues to be debated [28-30]. In order to determine if the intratumoral milieu could offer insights into the degree of clinical response to cytotoxic chemotherapy, histopathologic response to neoadjuvant chemotherapy was correlated to soluble mediator concentrations. Clinically, resected PC specimens with a poor histopathologic response to neoadjuvant therapy represent a group of treatment-resistant tumors. Indeed, significantly higher levels of the pro-inflammatory cytokines IL- $1 \beta$ and TNF $\alpha$ were observed in tumors from this population compared to tumors displaying a moderate to complete pathologic response to cytotoxic chemotherapy (Fig. 1). These preliminary data provide rationale for the continued evaluation of potential biologic mechanisms within the tumor microenvironment by which resistance to cytotoxic chemotherapy is maintained.

\section{Variations within the intratumoral inflammatory milieu correlate with clinicopathologic features}

In order to determine whether patterns of soluble mediator concentrations could offer further insights into the biology of PC, the inflammatory milieu was evaluated with respect to commonly used clinicopathologic parameters, such as positive lymph node ratio, serum CA 19-9 concentrations, tumor grade and tumor size. In our analysis of lymphatic metastasis using positive lymph node ratio, elevated 
Table 1 Inflammatory milieu within pancreatic tissue is predictive of malignancy

\begin{tabular}{|c|c|c|c|c|c|}
\hline & $\begin{array}{l}\text { Normal pancreas } \\
(n=6)\end{array}$ & $\begin{array}{l}\text { Pancreatitis } \\
(n=9)\end{array}$ & $\begin{array}{l}\text { Pancreatic cancer } \\
(n=36)\end{array}$ & $\begin{array}{l}P \text { value nonmalignant } \\
\text { vs. Pancreatic cancer }\end{array}$ & $\begin{array}{l}P \text { value pancreatitis } \\
\text { vs. Pancreatic cancer }\end{array}$ \\
\hline \multicolumn{6}{|c|}{ Growth factors } \\
\hline FGF-2 & $761(221)$ & $951(187)$ & $1674(177)$ & .009 & .063 \\
\hline PDGF-BB & $117(46)$ & $102(48)$ & $244(63)$ & .026 & .022 \\
\hline VEGF & $151(46)$ & $76(20)$ & $252(59)$ & .119 & .046 \\
\hline \multicolumn{6}{|c|}{ Chemokines } \\
\hline Eotaxin & $21.8(12.8)$ & $21.4(12.9)$ & $90.4(16.3)$ & $<.001$ & .002 \\
\hline Fractalkine & $55.1(12.8)$ & $43.0(9.4)$ & 71.1 (3.6) & .010 & .009 \\
\hline Gro & $163(67)$ & $463(296)$ & $531(87)$ & .007 & .043 \\
\hline $\mid \mathrm{P}-10$ & $69(33)$ & $70(32)$ & $620(148)$ & $<.001$ & $<.001$ \\
\hline MCP1 & 437 (136) & $378(104)$ & $1615(302)$ & $<.001$ & $<.001$ \\
\hline MCP3 & $4.7(1.9)$ & $2.5(0.8)$ & $16.6(2.8)$ & $<.001$ & $<.001$ \\
\hline MDC & $27(11)$ & $70(36)$ & $143(17)$ & $<.001$ & .009 \\
\hline MIP-1a & $14.3(4.9)$ & $16.9(7.6)$ & $37.9(4.6)$ & .001 & .009 \\
\hline MIP-1 $\beta$ & $22.1(9.4)$ & $14.3(5.0)$ & $38.4(6.1)$ & .007 & .008 \\
\hline RANTES & $766(261)$ & $1066(228)$ & $1929(235)$ & .032 & .178 \\
\hline \multicolumn{6}{|l|}{ Cytokines } \\
\hline GM-CSF & $4.1(2.0)$ & $1.4(0.3)$ & $14.0(3.9)$ & $<.001$ & $<.001$ \\
\hline IFNa2 & $13.0(2.7)$ & $8.7(2.3)$ & $19.9(2.4)$ & .013 & .012 \\
\hline IL-1a & $1.8(0.5)$ & $3.2(0.6)$ & $12.2(2.0)$ & $<.001$ & .003 \\
\hline IL-1RA & 157 (144) & $46(21)$ & $500(96)$ & $<.001$ & $<.001$ \\
\hline$I L-1 \beta$ & $0.6(0.1)$ & $0.9(0.1)$ & $1.3(0.2)$ & .032 & .262 \\
\hline IL-6 & $13.3(5.3)$ & $5.8(2.1)$ & 71.8 (23.3) & $<.001$ & $<.001$ \\
\hline $\mathrm{IL}-7$ & $3.9(1.0)$ & $4.5(1.0)$ & $10.4(0.8)$ & $<.001$ & $<.001$ \\
\hline IL-8 & 160 (154) & $61(37)$ & $848(161)$ & $<.001$ & .002 \\
\hline IL-15 & $1.7(0.2)$ & $2.0(0.3)$ & $3.5(0.4)$ & .001 & .021 \\
\hline TNFa & $1.6(0.7)$ & $1.4(0.4)$ & $3.3(0.4)$ & .002 & .010 \\
\hline
\end{tabular}

Concentrations expressed as mean (SE) in units of $\mathrm{pg} / \mathrm{mg}$ protein. All significant comparisons are shown for which $P<0.05$ using the Mann Whitney $U$ test

intratumoral EGF concentrations associated with a high degree of lymphatic metastasis $(\rho=0.332, P=.048)$, while high concentrations of IL-4 displayed the opposite trend, correlating with reduced lymphatic metastasis $(\rho=-0.377$;

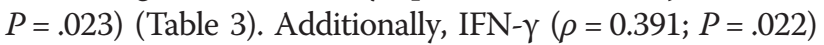
and RANTES $(\rho=0.475 ; P=.005)$ demonstrated significant positive correlations with serum CA 19-9 levels. High levels of IL- 8 and IP-10 associated with larger tumors ( $\rho=0.336 ; P=.042$ and $\rho=0.373 ; P=.023$ with respect

Table 2 Intratumoral milieu correlates with the administration of cytotoxic chemotherapy

\begin{tabular}{llll}
\hline & \multicolumn{2}{l}{ Neoadjuvant } & \\
\cline { 2 - 3 } & No $(n=26)$ & Yes $(n=10)$ & $P$ value \\
\hline Flt3L & $15.9(1.6)$ & $24.0(3.5)$ & .005 \\
IL-1a & $15.0(2.6)$ & $5.0(0.9)$ & .006 \\
IL-8 & $1129(197)$ & $114(35)$ & .003 \\
\hline
\end{tabular}

All significant comparisons are shown for which $P<0.05$ using the Mann Whitney U test to tumor size in $\mathrm{cm}$ ). The inflammatory milieu was then correlated with the degree of tumor differentiation observed in malignant tissue. Significant associations between poor tumor differentation and high concentrations of GMCSF, IL-15 and IL-8 were observed (Fig. 2). While these patterns provide insights into potential relationships between aspects of clinicopathological parameters and inflammation, these parameters do not always correlate with outcome.

We therefore aimed to determine if these known clinicopathologic predictors of outcome correlated with survival in our cohort. One commonly used predictor of overall survival in resected pancreatic cancer is the ratio of lymph nodes containing malignancy to the total number of lymph nodes resected and examined [31]. Indeed in our cohort, elevated positive lymph node ratios correlated strongly with reduced overall survival (HR 55.8; $P=.002$ ) (Table 4). This population therefore confirms the predictive nature of lymphatic metastasis. However, associations between 

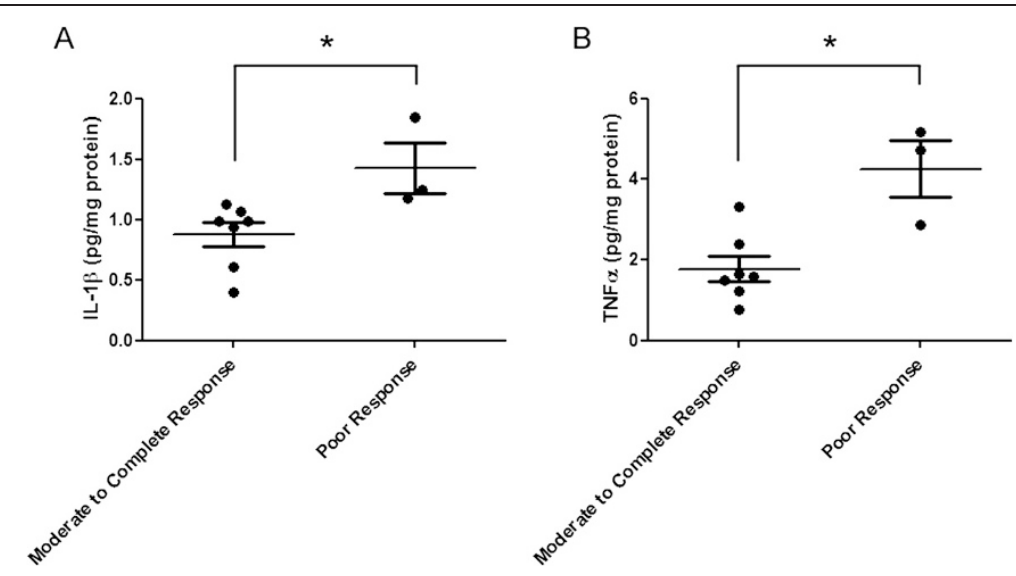

Fig. 1 Th1-associated cytokines within the tumor microenvironment correlate with treatment resistance in PC. Distributions are displayed comparing intratumoral concentrations of $\mathbf{a} I L-1 \beta$ and $\mathbf{b}$ TNFa in homogenates of pancreatic adenocarcinoma with histopathologic response to neoadjuvant chemotherapy. Bars represent mean values with standard error of the mean. ${ }^{*} P<0.05$ for each association using the Mann Whitney $\mathrm{U}$ test

survival and other commonly used prognostic parameters such as microscopically positive resection margins and poor tumor differentiation were not statistically significant in this cohort (HR 1.96; $P=.12$ and $\mathrm{HR}$ $2.28 ; P=.053$, respectively) (Table 4 ).

\section{Variations in the inflammatory milieu within the tumor microenvironment correlate with patient survival}

Since the common clinicopathological data were poor at predicting outcome, we next hypothesized that elements within the inflammatory milieu harbor superior prognostic value. The intratumoral milieu was correlated with overall survival in all 36 patients with PC who underwent surgical resection with curative intent. All patients had at least 6 months of clinical follow-up. Associations between the intratumoral milieu and overall survival were evaluated using both Kaplan-Meier and Cox proportional hazards models. Soluble mediators were first dichotomized using median concentrations and evaluated by logrank test in a Kaplan-Meier model. FGF-2, MDC, IL-4 and Flt-3 L significantly correlated with prolonged survival upon dichotomization (Table 5, Fig. 3). Due to the potential bias introduced from the artificial categorization of values dichotomized at the median, a proportional hazards

Table $\mathbf{3}$ Inflammatory milieu within the tumor microenvironment correlates with clinicopathologic parameters in PC specimens

\begin{tabular}{llll}
\hline Clinical parameter & Ligand & Spearman coefficient & $P$ value \\
\hline Positive Lymph Node Ratio & EGF & .332 & .048 \\
& IL-4 & -.377 & .023 \\
CA 19-9 (U/mL) & IFN-r & .391 & .022 \\
& RANTES & .475 & .005 \\
Tumor Size $(\mathrm{cm})$ & IL-8 & .336 & .045 \\
& IP-10 & .357 & .033
\end{tabular}

All significant comparisons are shown for which $P<0.05$ model was employed, allowing for direct correlation of intratumoral mediator concentration and survival. In this manner, high levels of both G-CSF and PDGF-AA correlated with reduced survival (HR 1.03; $P=.016$ and $H R$ $3.51 ; P=.012$, respectively) (Table 5).

Anecdotal observations of interest included: Four patients with tumors which demonstrated distinctly high PDGF-AA concentrations, at least double that of any other PC specimen, recurred within 6 months postoperatively. Further, two patients with tumors containing G-CSF concentrations over 5 times that of any other also recurred within 6 months, with one of these patients showing evidence of metastatic disease as soon as two months postoperatively. Conversely, two patients with tumors with undetectable MIP- $1 \alpha$ recurred within 6 months and three patients whose tumors had the highest intratumoral MIP- $1 \alpha$ concentrations were recurrence-free between one and three years postoperatively. Further, the patient with the highest intratumoral TNF $\alpha$ concentration remains recurrence-free 34 months postoperatively.

\section{Discussion}

Due to the dismal clinical outcomes associated with pancreatic adenocarcinoma and the continued debate surrounding therapeutic interventions, there is a tremendous need for the development of tools that can supplement current diagnostic and prognostic efforts. The extent of genetic and phenotypic heterogeneity specific to PC represents a major obstacle to the clinical application of developing biomarkers in PC. Efforts to further understand clinical observations regarding pathologic signaling within the tumor microenvironment have provided a novel focus that have led to major breakthroughs. Examples include therapeutic successes following Nab-paclitaxel infusion that is dependent upon SPARC expression in desmoplastic tumor-associated stroma [32, 33], and consistent 


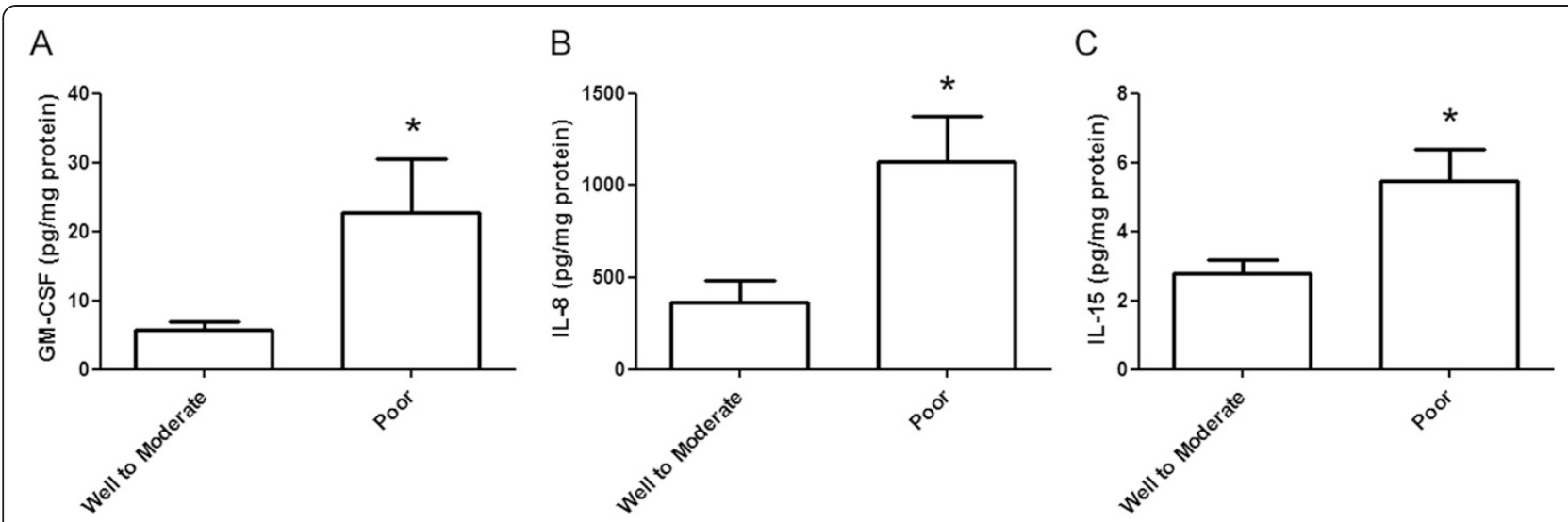

Tumor Differentiation

Fig. 2 Inflammatory milieu within the tumor microenvironment correlates with tumor grade. Intratumoral concentrations of a GM-CSF, b IL-8 and c IL-15 demonstrated significant correlations with high tumor grade. ${ }^{*} P<0.05$ for each association using the Mann Whitney $U$ test

observations that partial responses achieved from CD40 agonists led to the infiltration of tumoricidal macrophages into the local microenvironment $[34,35]$. In order to further understand clinically important paracrine signaling pathways within this local microenvironment, the work presented here has detected a unique inflammatory signature within pancreatic adenocarcinoma that is distinct from that of chronic, benign inflammation. Further, several members of this panel of markers were associated with specific clinicopathologic parameters, response to cytotoxic chemotherapy, and overall survival.

The almost universal development of treatment resistance and disease relapse following systemic cytotoxic or targeted therapies has made survival in PC achievable in only a small minority of patients. Mechanistically, chemoresistant phenotypes have been reproduced in vitro. However, the relevance of these findings to clinical practice

Table 4 Univariate analysis of overall survival

\begin{tabular}{lccc}
\hline & HR & $95 \% \mathrm{Cl}$ & $P$ value \\
\hline Age $(\mathrm{y})$ & 1.01 & $0.98-1.05$ & .556 \\
Neoadjuvant Therapy & 1.08 & $0.43-2.68$ & .870 \\
CA 19-9 (kU/mL) & 1.38 & $0.83-2.29$ & .215 \\
Major Vascular Resection & 2.66 & $0.85-8.30$ & .093 \\
R1 Resection & 1.96 & $0.84-4.53$ & .118 \\
Positive Lymph Node Ratio & 55.8 & $4.35-714$ & $.002^{*}$ \\
Poor Tumor Differentiation & 2.28 & $0.99-5.23$ & .053 \\
Tumor Size (cm) & 1.06 & $0.89-1.28$ & .506 \\
\hline
\end{tabular}

Clinicopathologic parameters were analyzed in a Cox proportional hazards model for 36 patients with surgically resected pancreatic adenocarcinoma. Abbreviations: $H R$ hazard ratio, $\mathrm{Cl}$ confidence interval, y years, $\mathrm{R} 1$ resection denotes a microscopically positive margin; Positive lymph node ratio refers to the number of lymph nodes positive for malignancy divided by the total number of lymph nodes examined; $\mathrm{cm}$ centimeters. ${ }^{*}, P<.05$ remains unclear. For example, gemcitabine resistance has been linked to the expression of gemcitabine-metabolizing proteins and DNA repair enzymes as well as the downregulation of nucleoside transporters. However, the clinical value of identifying these markers in resected PC specimens has yielded conflicting results [36]. Here we demonstrate that not only is the exposure to gemcitabine-based therapy associated with a different inflammatory milieu within the tumor, but also that differences in the milieu associate with the degree of clinical response, whereby increased levels of intratumoral IL1- $\beta$ and TNF- $\alpha$ are associated with poor histopathologic response to neoadjuvant therapy. These findings further support a wealth of investigations linking downstream NF- $\mathrm{kB}$ signaling to tumor progression and chemoresistance [37].

The observation that EGF levels correlated with the degree of lymph node metastasis is consistent with widespread evidence implicating EGF signaling in cancer progression and metastasis, culminating in a phase three trial employing EGFR inhibition in pancreatic cancer [3]. Conversely, high intratumoral concentrations of IL-4 displayed the opposite trend, correlating with reduced lymphatic metastasis, whereby patients with tumors high in IL-4 concentrations displayed roughly triple the survival of those with tumors expressing low levels of IL-4. In light of this finding it is important to note that direct stimulation of cancer cells with IL-4 generally results in augmented growth and proliferation [38-40]. However, this finding must be interpreted within the context of IL-4 signaling within the microenvironment. Indeed, constitutive IL-4 expressing cancers have demonstrated reduced growth in vivo due to the induction of a robust antitumor immune response [41]. Similarly, intratumoral levels of IL-8 and GM-CSF were predictive of tumor 
Table 5 Soluble mediators detected within the PC microenvironment correlate with prognosis

\begin{tabular}{|c|c|c|c|c|c|c|c|}
\hline & \multicolumn{3}{|c|}{ Dichotomized at median concentration } & \multicolumn{2}{|c|}{ Univariate PH model } & \multicolumn{2}{|c|}{ Multivariate PH model } \\
\hline & Median OS (Low) & Median OS (High) & $P$ value & $\mathrm{HR}(95 \% \mathrm{Cl})$ & $P$ value & $\mathrm{HR}(95 \% \mathrm{Cl})$ & $P$ value \\
\hline EGF & 10.4 & 11.0 & .165 & $1.23(0.98-1.54)$ & .078 & $1.10(.86-1.42)$ & .439 \\
\hline G-CSF & 18.3 & 7.5 & .064 & $1.03(1.01-1.06)$ & $.016^{*}$ & $1.02(1.00-1.05)$ & .071 \\
\hline PDGF-AA & 18.7 & 8.9 & .170 & $3.51(1.32-9.31)$ & $.012^{*}$ & $2.72(1.00-7.40)$ & $.050^{*}$ \\
\hline IL-6 & 11.0 & 10.4 & .500 & $12.0(0.85-172)$ & .066 & $6.10(0.39-96.13)$ & .198 \\
\hline FGF-2 & 7.1 & 20.7 & $.010^{*}$ & $0.60(0.37-0.97)$ & $.038^{*}$ & $0.59(0.37-0.93)$ & $.024^{*}$ \\
\hline TNFa & 7.5 & 18.3 & .085 & $0.78(0.63-0.98)$ & $.031^{*}$ & $0.79(0.64-0.97)$ & $.027^{*}$ \\
\hline MIP-1a & 10.4 & 14.9 & .352 & $0.98(0.96-1.00)$ & $.036^{*}$ & $0.98(0.97-1.00)$ & $.029 *$ \\
\hline $\mid \mathrm{L}-4$ & 7.6 & 20.7 & $.008^{*}$ & $0.85(0.70-1.04)$ & .112 & $0.26(0.75-1.08)$ & .902 \\
\hline Flt-3 L & 7.1 & 18.7 & $.037^{*}$ & $0.95(0.88-1.01)$ & .103 & $0.94(0.86-1.02)$ & .113 \\
\hline MDC & 7.6 & 20.7 & $.049^{*}$ & $0.01(0-1.37)$ & .065 & $0.01(0-2.24)$ & .098 \\
\hline Eotaxin & 7.5 & 18.7 & .055 & $0.03(0-15.2)$ & .263 & $0.09(0-47.9)$ & .454 \\
\hline
\end{tabular}

Soluble mediators were dichotomized at median concentrations and survival was evaluated using Kaplan-Meier analysis with $P$ values determined using the log-rank test (left). Associations between soluble mediator concentrations and survival were then evaluated in a continuous fashion using a Cox proportional hazards model (middle). Finally, concentrations were evaluated in a multivariate Cox proportional hazards model with positive lymph node ratio (right). Abbreviations: $\mathrm{PH}$ proportional hazards, OS overall survival, $\mathrm{HR}$ hazard ratio, $\mathrm{Cl}$ confidence interval. ${ }^{*}, P<.05$

grade while IL-8 levels were also positively associated with tumor size. Again this is consistent with previous findings that suggest that IL-8 and GM-CSF produced in the tumor microenvironment promote immune evasion in PC [42-44]. Interestingly, the administration of cytotoxic chemotherapy was strongly associated with significantly lower intratumoral IL-8 concentrations. The investigation of the intratumoral inflammatory milieu has therefore revealed consistent correlations between IL-8 concentrations, histopathologic findings and the administration of chemotherapy.

As alluded to above, several of these mediators could also be used to predict survival. For instance, high intratumoral G-CSF levels correlated with reduced overall survival, which is supported in literature relating myeloidderived suppressor cell infiltration to tumor progression and angiogenesis [45]. Of particular interest is the prolonged survival observed in patients with high intratumoral FGF-2, known to stimulate fibroblast migration, wound healing and generally thought to be a growth factor which supports growing tumors. However, it is generally accepted that FGF-2 is abundant in most tissues, concentrated in basement membranes and at cell surfaces in inactive forms. Tissue injury leads to FGF-2 activation and subsequent promotion of wound healing processes known to promote tumor growth, invasion and angiogenesis [46]. In this context, reduced FGF-2 concentrations in tissue homogenates may paradoxically reflect increased FGF-2 activation, which would lead to the expected findings of reduced survival.

The inability to follow the intratumoral inflammatory milieu over time represents a significant limitation to this type of analysis, as this information will be critical to elucidating the relationship between local inflammation and treatment strategies in PC. In addition, stratification of long-term survival into treatment-naïve and treatmentexposed tumors will be essential in validating these relationships. However, this analysis currently lacks the power to dichotomize in this fashion. While grouping mediators into functionally relevant categories may address our current lack of power, the pleiotropic nature of these soluble mediators may lead to improper interpretations in the absence of functional analyses. Further, it has not escaped our notice that VEGF demonstrated no correlation with survival in this analysis. The extensive body of work associating VEGF signaling with angiogenesis and tumor progression has led many groups to investigate potential correlations between VEGF expression and survival in PC. Subsequent analyses have yielded conflicting results [47-49]. Importantly, this is not the first clinical cohort to demonstrate a nonsignificant correlation between intratumoral VEGF levels and overall survival in PC.

\section{Conclusions}

In summary, pancreatic adenocarcinoma is a devastating malignancy with an extremely poor prognosis. High ratios of tumor stroma to cancer cells plague the sensitivity of cytologic diagnosis of PC; In fact, even direct pathologic analysis of $\mathrm{PC}$ biopsies can yield inconsistent results with high interobserver variability [50]. Highly specific, reliable biochemical signatures obtained from these small samples that improve diagnostic sensitivity could dramatically improve the clinical care of PC patients. Further, treatment algorithms for PC in the absence of metastasis are currently anatomic based and lack attention to variations in biology. Thus, there is further need to develop accurate biomarkers capable of predicting response to systemic therapies or the futility of surgical or radiation therapy. 


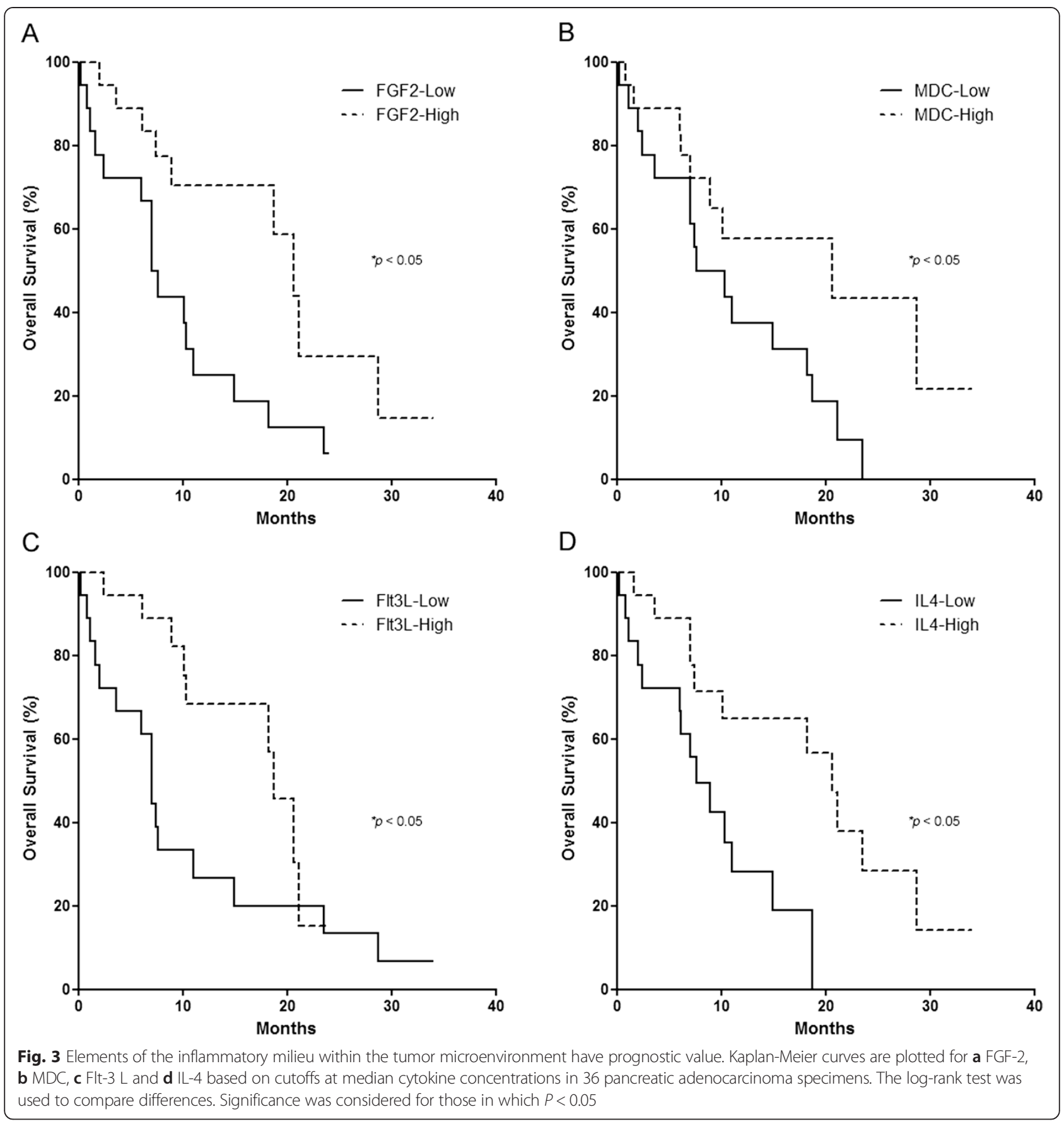

Unfortunately, the current literature is characterized by marked variability between individual studies as to the relative prognostic impact of several biomarkers in PC. Here, in contrast to studies that evaluated these properties using a single or a couple biomarkers, we have identified novel relationships between tissue examination and clinical outcome by quantitatively evaluating the milieu of the tumor microenvironment utilizing fresh pancreatic surgical specimens. It is important to note that even though some of these associations are counterintuitive based on the currently understood biology, the greater context and complexity of the tumor microenvironment in PC is not currently appreciated. Thus, these data combined with a better understanding of the context-dependence of inflammatory signaling, may eventually offer the opportunity to identify patterns that improve interpretations in cancer and emphasize the importance of investigating the tumor microenvironment as a whole. Nonetheless, results 
presented here exhibit a high degree of reproducibility and provide rationale to prospectively evaluate these markers as diagnostic and prognostic tools.

\section{Competing interests}

The authors declare that they have no competing interests.

\section{Authors' contributions}

$\mathrm{BB}, \mathrm{HL}$ and $\mathrm{AK}$ participated in the experimental design and data analysis. RT, GS, CL, TG, KB and LM assisted with the statistical analysis and provided a multidisciplinary foundation necessary for careful interpretation. DD, JT, SW and $\mathrm{SH}$ drafted and revised the manuscript. All authors have read and approved the final copy of the manuscript.

\section{Acknowledgements}

This research was supported by funding from the NIH (NCI 5T32CA106493-09) as well as the Cracchiolo Foundation.

\begin{abstract}
Author details
${ }^{1}$ Department of Surgery, College of Medicine, University of Florida Health Science Center, Room 6116, Shands Hospital, 1600 SW Archer Rd, Gainesville, FL 32610, USA. ${ }^{2}$ Department of Oral Biology, College of Dentistry, University of Florida Health Science Center, Gainesville, FL 32610, USA. ${ }^{3}$ North Florida/ South Georgia Veterans Health System, Department of Surgery, University of Florida College of Medicine, Gainesville, FL 32610, USA. ${ }^{4}$ Department of Pathology, College of Medicine, University of Florida Health Science Center, Gainesville, FL 32610, USA. 'Department of Medicine, College of Medicine, University of Florida Health Science Center, Gainesville, FL 32610, USA
\end{abstract}

\section{Received: 4 May 2015 Accepted: 16 October 2015}

\section{Published online: 24 October 2015}

\section{References}

1. Burris 3rd HA, Moore MJ, Andersen J, Green MR, Rothenberg ML, Modiano $M R$, et al. Improvements in survival and clinical benefit with gemcitabine as first-line therapy for patients with advanced pancreas cancer: a randomized trial. J Clin Oncol. 1997;15(6):2403-13.

2. Conroy T, Desseigne F, Ychou M, Bouche O, Guimbaud R, Becouarn Y, et al. FOLFIRINOX versus gemcitabine for metastatic pancreatic cancer. N Engl J Med. 2011;364(19):1817-25.

3. Moore MJ, Goldstein D, Hamm J, Figer A, Hecht JR, Gallinger S, et al. Erlotinib plus gemcitabine compared with gemcitabine alone in patients with advanced pancreatic cancer: a phase III trial of the National Cancer Institute of Canada Clinical Trials Group. J Clin Oncol. 2007;25(15):1960-6.

4. Wolf AM, Pucci MJ, Gabale SD, McIntyre CA, Irizarry AM, Kennedy EP, et al. Safety of perioperative aspirin therapy in pancreatic operations. Surgery. 2014;155(1):39-46.

5. Rahib L, Smith BD, Aizenberg R, Rosenzweig AB, Fleshman JM, Matrisian LM. Projecting cancer incidence and deaths to 2030: the unexpected burden of thyroid, liver, and pancreas cancers in the United States. Cancer Res. 2014;74(11):2913-21.

6. Muerkoster S, Wegehenkel K, Arlt A, Witt M, Sipos B, Kruse ML, et al. Tumor stroma interactions induce chemoresistance in pancreatic ductal carcinoma cells involving increased secretion and paracrine effects of nitric oxide and interleukin-1 beta. Cancer Res. 2004;64(4):1331-7.

7. Hausmann S, Kong B, Michalski C, Erkan M, Friess H. The role of inflammation in pancreatic cancer. Adv Exp Med Biol. 2014;816:129-51.

8. Kadaba R, Birke H, Wang J, Hooper S, Andl CD, Di Maggio F, et al. Imbalance of desmoplastic stromal cell numbers drives aggressive cancer processes. J Pathol. 2013;230(1):107-17.

9. Vonlaufen A, Joshi S, Qu C, Phillips PA, Xu Z, Parker NR, et al. Pancreatic stellate cells: partners in crime with pancreatic cancer cells. Cancer Res. 2008;68(7):2085-93.

10. Koay EJ, Truty MJ, Cristini V, Thomas RM, Chen R, Chatterjee D, et al. Transport properties of pancreatic cancer describe gemcitabine delivery and response. J Clin Invest. 2014:124(4):1525-36.

11. Galon J, Costes A, Sanchez-Cabo F, Kirilovsky A, Mlecnik B, Lagorce-Pages C, et al. Type, density, and location of immune cells within human colorectal tumors predict clinical outcome. Science. 2006;313(5795):1960-4.
12. Zhang L, Conejo-Garcia JR, Katsaros D, Gimotty PA, Massobrio M, Regnani G, et al. Intratumoral T cells, recurrence, and survival in epithelial ovarian cancer. N Engl J Med. 2003;348(3):203-13.

13. Naito Y, Saito K, Shiiba K, Ohuchi A, Saigenji K, Nagura H, et al. CD8+ T cells infiltrated within cancer cell nests as a prognostic factor in human colorectal cancer. Cancer Res. 1998;58(16):3491-4.

14. Chatterjee D, Katz MH, Rashid A, Estrella JS, Wang H, Varadhachary GR, et al. Pancreatic intraepithelial neoplasia and histological changes in nonneoplastic pancreas associated with neoadjuvant therapy in patients with pancreatic ductal adenocarcinoma. Histopathology. 2013;63(6):841-51.

15. Fukunaga A, Miyamoto M, Cho Y, Murakami S, Kawarada Y, Oshikiri T, et al. CD8+ tumor-infiltrating lymphocytes together with CD4+ tumor-infiltrating lymphocytes and dendritic cells improve the prognosis of patients with pancreatic adenocarcinoma. Pancreas. 2004;28(1):e26-31.

16. Bang UC, Benfield T, Hyldstrup L, Bendtsen F, Beck Jensen JE. Mortality, cancer, and comorbidities associated with chronic pancreatitis-a Danish nationwide matched-cohort study. Gastroenterology. 2013;146(4):989-94.

17. Gillies RJ, Verduzco D, Gatenby RA. Evolutionary dynamics of carcinogenesis and why targeted therapy does not work. Nat Rev Cancer. 2012;12(7):487-93.

18. Chopra M, Lang I, Salzmann S, Pachel C, Kraus S, Bauerlein CA, et al. Tumor necrosis factor induces tumor promoting and anti-tumoral effects on pancreatic cancer via TNFR1. PLoS One. 2013;8(9):e75737.

19. Means AL, Meszoely IM, Suzuki K, Miyamoto Y, Rustgi AK, Coffey Jr RJ, et al. Pancreatic epithelial plasticity mediated by acinar cell transdifferentiation and generation of nestin-positive intermediates. Development. 2005;132(16):3767-76.

20. Ellenrieder V, Hendler SF, Ruhland C, Boeck W, Adler G, Gress TM. TGF-betainduced invasiveness of pancreatic cancer cells is mediated by matrix metalloproteinase-2 and the urokinase plasminogen activator system. Int J Cancer. 2001:93(2):204-11.

21. Greco E, Basso D, Fogar P, Mazza S, Navaglia F, Zambon CF, et al. Pancreatic cancer cells invasiveness is mainly affected by interleukin-1 beta not by transforming growth factor-beta1. Int J Biol Markers. 2005;20(4):235-41.

22. Melisi D, Niu J, Chang Z, Xia Q, Peng B, Ishiyama S, et al. Secreted interleukin-1alpha induces a metastatic phenotype in pancreatic cancer by sustaining a constitutive activation of nuclear factor-kappaB. Mol Cancer Res. 2009;7(5):624-33.

23. Miyamoto Y, Hosotani R, Doi R, Wada M, Ida J, Tsuji S, et al. Interleukin-6 inhibits radiation induced apoptosis in pancreatic cancer cells. Anticancer Res. 2001;21(4A):2449-56.

24. Bellone G, Smirne C, Mauri FA, Tonel E, Carbone A, Buffolino A, et al. Cytokine expression profile in human pancreatic carcinoma cells and in surgical specimens: implications for survival. Cancer Immunol Immunother. 2006:55(6):684-98.

25. Takamori H, Oades ZG, Hoch OC, Burger M, Schraufstatter IU. Autocrine growth effect of IL-8 and GROalpha on a human pancreatic cancer cell line, Capan-1. Pancreas. 2000;21(1):52-6.

26. Rhim AD, Oberstein PE, Thomas DH, Mirek ET, Palermo CF, Sastra SA, et al. Stromal Elements Act to Restrain, Rather Than Support, Pancreatic Ductal Adenocarcinoma. Cancer Cell. 2014;25(6):735-47.

27. Evans DB, Rich TA, Byrd DR, Cleary KR, Connelly JH, Levin B, et al. Preoperative chemoradiation and pancreaticoduodenectomy for adenocarcinoma of the pancreas. Arch Surg. 1992;127(11):1335-9.

28. Breslin TM, Hess KR, Harbison DB, Jean ME, Cleary KR, Dackiw AP, et al. Neoadjuvant chemoradiotherapy for adenocarcinoma of the pancreas: treatment variables and survival duration. Ann Surg Oncol. 2001;8(2):123-32.

29. Le Scodan R, Mornex F, Partensky C, Mercier C, Valette PJ, Ychou M, et al. Histopathological response to preoperative chemoradiation for resectable pancreatic adenocarcinoma: the French Phase II FFCD 9704-SFRO Trial. Am J Clin Oncol. 2008;31(6):545-52.

30. White RR, Xie HB, Gottfried MR, Czito BG, Hurwitz HI, Morse MA, et al. Significance of histological response to preoperative chemoradiotherapy for pancreatic cancer. Ann Surg Oncol. 2005;12(3):214-21.

31. La Torre M, Nigri G, Petrucciani N, Cavallini M, Aurello P, Cosenza G, et al. Prognostic assessment of different lymph node staging methods for pancreatic cancer with $\mathrm{RO}$ resection: $\mathrm{pN}$ staging, lymph node ratio, log odds of positive lymph nodes. Pancreatology. 2014;14(4):289-94.

32. Infante JR, Matsubayashi $H$, Sato N, Tonascia J, Klein AP, Riall TA, et al. Peritumoral fibroblast SPARC expression and patient outcome with resectable pancreatic adenocarcinoma. J Clin Oncol. 2007;25(3):319-25. 
33. Von Hoff DD, Ramanathan RK, Borad MJ, Laheru DA, Smith LS, Wood TE, et al. Gemcitabine plus nab-paclitaxel is an active regimen in patients with advanced pancreatic cancer: a phase I/II trial. J Clin Oncol. 2011;29(34):4548-54.

34. Beatty GL, Chiorean EG, Fishman MP, Saboury B, Teitelbaum UR, Sun W, et al. CD40 agonists alter tumor stroma and show efficacy against pancreatic carcinoma in mice and humans. Science. 2011;331(6024):1612-6.

35. Luheshi N, Davies $\mathrm{G}$, Legg J. Understanding the influence of the tumor microenvironment on macrophage responses to CD40 agonists. Oncoimmunology. 2014;3(1):e27615.

36. Costello E, Greenhalf W, Neoptolemos JP. New biomarkers and targets in pancreatic cancer and their application to treatment. Nat Rev Gastroenterol Hepatol. 2012;9(8):435-44.

37. Karin M. Nuclear factor-kappaB in cancer development and progression. Nature. 2006:441(7092):431-6

38. Roca H, Craig MJ, Ying C, Varsos ZS, Czarnieski P, Alva AS, et al. IL-4 induces proliferation in prostate cancer $\mathrm{PC} 3$ cells under nutrient-depletion stress through the activation of the JNK-pathway and survivin up-regulation. J Cell Biochem. 2012;113(5):1569-80.

39. Lee SO, Pinder E, Chun JY, Lou W, Sun M, Gao AC. Interleukin-4 stimulates androgen-independent growth in LNCaP human prostate cancer cells. Prostate. 2008:68(1):85-91.

40. Prokopchuk O, Liu Y, Henne-Bruns D, Kornmann M. Interleukin-4 enhances proliferation of human pancreatic cancer cells: evidence for autocrine and paracrine actions. Br J Cancer. 2005:92(5):921-8.

41. Eguchi J, Hiroishi K, Ishii S, Baba T, Matsumura T, Hiraide A, et al. Interleukin4 gene transduced tumor cells promote a potent tumor-specific Th1-type response in cooperation with interferon-alpha transduction. Gene Ther. 2005:12(9):733-41.

42. Mace TA, Ameen Z, Collins A, Wojcik S, Mair M, Young GS, et al. Pancreatic cancer-associated stellate cells promote differentiation of myeloid-derived suppressor cells in a STAT3-dependent manner. Cancer Res. 2013;73(10):3007-18.

43. Pylayeva-Gupta Y, Lee KE, Hajdu CH, Miller G, Bar-Sagi D. Oncogenic Kras-induced GM-CSF production promotes the development of pancreatic neoplasia. Cancer Cell. 2012;21(6):836-47.

44. Xie K. Interleukin-8 and human cancer biology. Cytokine Growth Factor Rev. 2001:12(4):375-91

45. Shojaei F, Wu X, Qu X, Kowanetz M, Yu L, Tan M, et al. G-CSF-initiated myeloid cell mobilization and angiogenesis mediate tumor refractoriness to anti-VEGF therapy in mouse models. Proc Natl Acad Sci. 2009;106(16):6742-7.

46. Kato M, Wang H, Kainulainen V, Fitzgerald ML, Ledbetter S, Ornitz DM, et al, Physiological degradation converts the soluble syndecan-1 ectodomain from an inhibitor to a potent activator of FGF-2. Nat Med. 1998;4(6):691-7.

47. Smith RA, Tang J, Tudur-Smith C, Neoptolemos JP, Ghaneh P. Meta-analysis of immunohistochemical prognostic markers in resected pancreatic cancer. Br J Cancer. 2011;104(9):1440-51.

48. Jamieson NB, Carter CR, McKay CJ, Oien KA. Tissue biomarkers for prognosis in pancreatic ductal adenocarcinoma: a systematic review and metaanalysis. Clin Cancer Res. 2011;17(10):3316-31.

49. Ansari D, Rosendahl A, Elebro J, Andersson R. Systematic review of immunohistochemical biomarkers to identify prognostic subgroups of patients with pancreatic cancer. Br J Surg. 2011;98(8):1041-55.

50. Larghi A, Correale L, Ricci R, Abdulkader I, Monges G, Iglesias-Garcia J, et al. Interobserver agreement and accuracy of preoperative endoscopic ultrasound-guided biopsy for histological grading of pancreatic cancer. Endoscopy. 2014;47(4):308-14.

\section{Submit your next manuscript to BioMed Central and take full advantage of:}

- Convenient online submission

- Thorough peer review

- No space constraints or color figure charges

- Immediate publication on acceptance

- Inclusion in PubMed, CAS, Scopus and Google Scholar

- Research which is freely available for redistribution 